\title{
Emittance measurement along transport beam line for laser driven protons
}

\author{
M. J. Wu๑,${ }^{1}$ D. Y. Li,${ }^{1}$ J. G. Zhu, ${ }^{1}$ T. Yang $\odot,{ }^{1}$ X. Y. Hu, ${ }^{1}$ Y. X. Geng,,${ }^{1}$ K. Zhu, ${ }^{1}$ M. J. Easton $\odot,{ }^{1}$ \\ Y. Y. Zhao, ${ }^{1}$ A. L. Zhang, ${ }^{1}$ H. Y. Lu, ${ }^{1}$ W. J. Ma, ${ }^{1}$ C. Lin, ${ }^{1,2,{ }^{*}}$ and X. Q. Yan ${ }^{1,2, \dagger}$ \\ ${ }^{1}$ State Key Laboratory of Nuclear Physics and Technology, and Key Laboratory of HEDP of the \\ Ministry of Education, CAPT, Peking University, Beijing 100871, China \\ ${ }^{2}$ Collaborative Innovation Center of Extreme Optics, Shanxi University, Shanxi 030006, China
}

(Received 9 January 2020; accepted 2 March 2020; published 11 March 2020)

\begin{abstract}
Particle emittance quantifies the ability to focus and transport a beam, and is one of the most important parameters for a beamline. The emittance of a proton beam produced by ultraintense laser irradiation of a micron-thick flat solid target has been measured systematically for the first time, using three different methods at different positions along the transport beamline: pepper-pot method, quadrupole triplet scan technique, and single-shot emittance measurement. Emittance growth is shown both in experiments and in simulations using CST. An over 3-fold emittance growth was found for $5 \mathrm{MeV}$ laser-driven protons with an energy spread of $\pm 2 \%$ and divergence of $\pm 20 \mathrm{mrad}$ after being transported $5.9 \mathrm{~m}$ in the experiment, due to the energy spread and angular dispersion of the protons.
\end{abstract}

DOI: $10.1103 /$ PhysRevAccelBeams.23.031302

\section{INTRODUCTION}

Recently the energy of protons accelerated by ultrahighintensity laser pulses irradiating solid ultrathin targets has reached nearly $100 \mathrm{MeV}$ within acceleration distances of tens of micrometers [1]. The low emittance [2], tiny source size [3], and short pulse duration of the proton beam [4,5] shows great potential for novel scientific and engineering applications, such as material science [6,7], imaging [8], cancer treatment [9], inertial confinement fusion [10], and warm dense matter $[11,12]$. On the other hand, the proton beam is also characterized by broad energy spread and divergence angle. Proper guidance and transportation are therefore necessary [13-16], which should be based on a better characterization of such accelerated beams. The emittance is a measure derived from the beam distribution in phase space that quantifies its divergence and ability to be focused. Hence it is of great importance to measure the emittance of laser-accelerated protons during transportation.

The Target Normal Sheath Acceleration (TNSA) method produces an electrostatic sheath field upwards of $10^{12} \mathrm{~V} / \mathrm{m}$ that ionizes the surface atoms almost instantaneously, forming a few-nanometer-thick ion layer, with the electron sheath resembling a virtual cathode [17,18].

\footnotetext{
*1c0812@pku.edu.cn

xueqingyan@pku.edu.cn
}

Published by the American Physical Society under the terms of the Creative Commons Attribution 4.0 International license. Further distribution of this work must maintain attribution to the author(s) and the published article's title, journal citation, and DOI.
The accelerated protons, which originate primarily from contaminant layers of water vapor or hydrocarbons on the target surface, have an exponentially decaying energy spectrum and a divergence angle of around $10^{\circ}$ [19]. Microstructure targets have been used to image the initial accelerating sheath and to fully reconstruct the proton transverse phase space, showing that the normalized rootmean-square (rms) emittance from the target is as low as $<0.004 \mathrm{~mm}$ mrad for proton with energy $>10 \mathrm{MeV}$, which is 100 times smaller than typical rf accelerators [2]. However, the emittance measured by the microstructure target may be different from a flat target, due to the filamentation of electrons and the change of the sheath field [3]. Attempts have been made to measure the beam emittance using projection, on a distant film, of objects such as slits [20] or meshes [4], showing a value for normalized emittance between 0.1 and $0.2 \mathrm{~mm}$ mrad. However, the beam does not propagate ballistically due to space charge, and objects placed in the beam path may collect charge [2], which deflects the beam and causes measurement errors. In the subsequent propagation of the beam, or in a transport process in a beamline, further emittance growth can be produced by energy chromatic aberrations, as well as nonlinearity in applied forces [21].

In this paper, we systematically study the emittance growth caused by the phase-space distribution diffusion of protons during the transport, and present the first experimental emittance measurement of a laser-accelerated proton beam through a beamline. Three methods are employed: the pepper-pot method [22], the quadrupole scan technique (QST) [23], and a single-shot emittance measurement with a dipole magnet [24]. We show that, for laser-accelerated $\mathrm{MeV}$-energy protons, the normalized rms 
emittance is preserved at a level of a percent of $\mathrm{mm} \cdot \mathrm{mrad}$ even under the chromatic effect and the higher-order field away from the axis of the magnets [21].

\section{EMITTANCE}

Two kinds of emittance are used in this paper and measured in this experiment, following Floettmann [25]. The first one is the normalized rms emittance derived in the phase space $\left(x, p_{x}\right)$, defined as:

$$
\begin{aligned}
\varepsilon_{n, \mathrm{rms}} & =\sqrt{\left\langle x^{2}\right\rangle\left\langle\beta^{2} \gamma^{2} x^{\prime 2}\right\rangle-\left\langle x \beta \gamma x^{\prime}\right\rangle^{2}} \\
& =\frac{1}{m_{0} c} \sqrt{\left\langle x^{2}\right\rangle\left\langle p_{x}^{2}\right\rangle-\left\langle x p_{x}\right\rangle^{2}},
\end{aligned}
$$

where relativistic velocity $\beta=v / c$ and $\gamma$ is the relativistic factor of the protons, $x^{\prime}=p_{x} / p_{z}$ is the divergence angle of proton, $m_{0}$ is the rest mass of proton and $\langle\cdots\rangle$ denotes the second central moment of the distribution. This normalized rms emittance $\varepsilon_{n, \text { rms }}$ is related to the area of the phase space ellipse. In the presence of a significant energy spread, $\varepsilon_{n, \text { rms }}$ will grow in a drift space due to the correlation of the transverse particle position and the longitudinal particle momentum $[25,26]$.

To measure $\varepsilon_{n \text {,rms }}$ in an experiment, the method must have both energy and spatial resolution, such as the signal shot measurement after an energy-dispersion component like a dipole magnet [24]. For an emittance measurement which only gives the angular and spatial distribution, like the pepper-pot method, what is actually measured is the normalized trace space rms emittance, in the trace space $\left(x, x^{\prime}\right)$, defined as:

$$
\begin{aligned}
\varepsilon_{n, \mathrm{r}, \mathrm{rms}} & =\frac{\bar{p}_{z}}{m_{0} c} \sqrt{\left\langle x^{2}\right\rangle\left\langle x^{\prime 2}\right\rangle-\left\langle x x^{\prime}\right\rangle^{2}} \\
& =\frac{\bar{p}_{z}}{m_{0} c} \sqrt{\left\langle x^{2}\right\rangle\left\langle\frac{p_{x}}{p_{z}}\right\rangle^{2}-\left\langle x \frac{p_{x}}{p_{z}}\right\rangle^{2}},
\end{aligned}
$$

where $\bar{p}_{z}$ denotes the average longitudinal momentum.

The trace space emittance $\varepsilon_{n, \text { tr.rms }}$ is only correlated with the beam geometry. Both $\varepsilon_{n, \text { rms }}$ and $\varepsilon_{n, \text { tr rms }}$ will vary in the presence of a magnet, especially when the beam divergence angle is large enough to exceed the acceptance envelope or the linear magnetic field region.

This emittance measurement experiment was carried out at the Compact Laser Plasma Accelerator (CLAPA) at Peking University [13]. Figure 1 shows a schematic of the experiment. An $f / 3.5$ off-axis parabola (OAP) mirror focuses the laser pulse with $30 \mathrm{fs}$ duration and $800 \mathrm{~nm}$ wavelength to a focal spot of $5 \mu \mathrm{m}$ diameter full-width-athalf-maximum (FWHM), containing 30\% of total laser energy of $1.3 \mathrm{~J}$, corresponding to an intensity of about $6 \times 10^{19} \mathrm{~W} / \mathrm{cm}^{2}$. The laser pluses are incident onto 5 -micron-thick plastic foils at $30^{\circ}$ with respect to the target

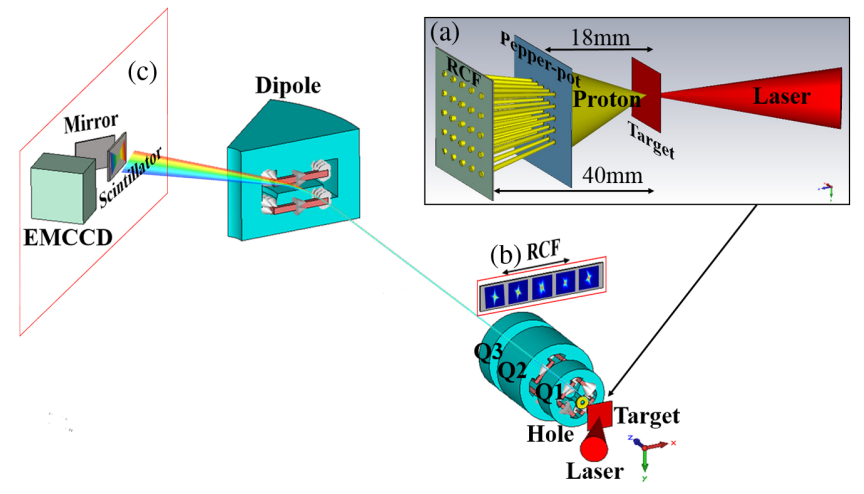

FIG. 1. Setup of the emittance measurement experiment on the laser proton beam line. Protons are accelerated along the $z$ direction and focused by the electromagnetic quadrupole triplet lens, in which Q1 and Q3 focus in the $x$-direction and Q2 focuses in the $y$-direction. Three methods were employed for the emittance measurement: (a) Pepper-pot; (b) QST, where the focal spot of the proton beam was detected by a RCF stack composed by an aluminum foil and one EBT film; and (c) singleshot emittance measurement after the dipole.

normal direction. The originally accelerated TNSA proton beams have exponential decaying energy distribution with a cutoff energy around $6.5 \mathrm{MeV}$ [21] and divergence of hundreds of mrad [19]. The protons are primarily focused by a quadrupole triplet electromagnet with an entrance at $14 \mathrm{~cm}$ behind the target, and then energy dispersed by a $45^{\circ}$ dipole magnet [27] placed $1.2 \mathrm{~m}$ behind the triplet. Three methods were employed: (a) pepper-pot right after the target, (b) quadrupole scan technique using the magnet triplet and (c) single-shot emittance measurement with the dipole magnet. Details of each measurement are as follows.

\section{PEPPER-POT}

A pepper-pot mask was placed $12 \mathrm{~mm}$ after the target, which was made of molybdenum with a $27 \times 27$ array of holes with $0.15 \mathrm{~mm}$ diameter and $0.75 \mathrm{~mm}$ interval. The protons passing through each hole form a beamlet with a certain divergence angle. A radio-chromic film (RCF) detector (EBT3) covered by a $90 \mu \mathrm{m}$ aluminum foil was placed $28 \mathrm{~mm}$ downstream of the pepper-pot to record the profile of the beamlets. The hole diameter and the distance between pepper-pot and RCF have been carefully designed to keep the diameter of each beamlet almost constant before detection, to avoid the space charge effect. The EBT RCF is composed by two substrate layers with the same thickness of $125 \mu \mathrm{m}$ and one sensitive layer with a thickness of $30 \mu \mathrm{m}$ in between. Then we put the same parameters in a Monte Carlo program SRIM (The Stopping and Range of Ions in Matter) [28] and calculated the trajectory and energy deposition of protons with different energies through the $90 \mu \mathrm{m}$ thickness $\mathrm{Al}$ foil and the EBT RCF. Since the Bragg Peak of proton with $4.9 \mathrm{MeV}$ energy is just on the front surface of the sensitive layer, and the Bragg 


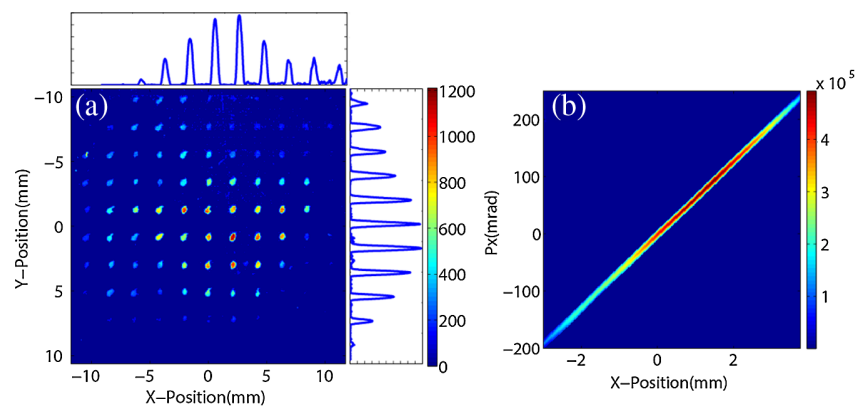

FIG. 2. Experimental results using the pepper-pot method. (a) Raw data from the RCF detector; detected proton energy is $(5.0 \pm 0.1) \mathrm{MeV}$. (b) Interpolated phase space diagram. Color scale is the proton count.

Peak of proton with $5.1 \mathrm{MeV}$ is on the back surface of the sensitive layer, we define the central proton energy detected by the EBT RCF is $5 \mathrm{MeV}$ with an energy spread of $\pm 2 \%$. In this monoenergetic case, $\varepsilon_{n, \mathrm{rms}} \approx \varepsilon_{n \text {,tr,rms }}$, and can be calculated as [22]:

$$
\begin{aligned}
\varepsilon_{n, \mathrm{rms}}^{2} & \approx \frac{\beta^{2} \gamma^{2}}{N^{2}}\left\{\left[\sum_{j=1}^{p} n_{j}\left(x_{\mathrm{s} j}-\bar{x}\right)^{2}\right]\left[\sum_{j=1}^{p}\left[n_{j} \sigma_{x_{j}^{\prime}}^{2}+n_{j}\left(\overline{x_{j}^{\prime}}-\overline{x^{\prime}}\right)^{2}\right]\right]\right. \\
& \left.-\left[\sum_{j=1}^{p} n_{j} x_{\mathrm{s} j} \bar{x}_{j}^{\prime}-N \bar{x} \bar{x}^{\prime}\right]^{2}\right\}
\end{aligned}
$$

where $N$ is the proton number detected by the RCF, $n_{j}$ is the number of particles passing through $j$ th slit, which is a weighting of spot intensity, $x_{\mathrm{s} j}$ is the $j$ th slit's position, and $p$ is the total number of slits. $\bar{x}$ and $\overline{x^{\prime}}$ are the mean position and divergence of all beamlets. $\bar{x}_{j}^{\prime}$ and $\sigma_{x_{j}^{\prime}}$ are the mean position and rms divergence of $j$ th beamlet. The measured microbeam spots and the phase space reconstructed from pepper-pot method are shown in Fig. 2(a) and (b), indicating that the beam has a divergence angle of $\pm 200 \mathrm{mrad}$ and a laminar distribution. We used a linear interpolation the measured values for all the protons $( \pm 200 \mathrm{mrad})$ to calculate the emittance value. The measured emittance $\varepsilon_{n, \text { tr rms }}$ is $0.8 \mathrm{~mm} \mathrm{mrad}$ for the full beam $( \pm 200 \mathrm{mrad})$, and $<0.02 \mathrm{~mm}$ mrad for protons with divergence smaller than $\pm 20 \mathrm{mrad}$.

\section{QUADRUPOLE SCAN TECHNIQUE (QST)}

Next, the pepper-pot was removed and the accelerated protons were collected by the electromagnetic quadrupole triplet, as shown in Fig. 1(b), where Q1 and Q3 focus horizontally (x-direction) and Q2 focuses vertically (y-direction) [13,27]. In order to reduce the effect of nonlinear forces and edge fields, a $6 \mathrm{~mm}$-diameter hole (corresponding to $\pm 20 \mathrm{mrad}$ ) is placed at the entrance of Q1 to satisfy the paraxial approximation. The beam profile was recorded $2.5 \mathrm{~m}$ away from $\mathrm{Q} 3$ using an $\mathrm{RCF}$ detector,
TABLE I. Triplet parameters.

\begin{tabular}{lccc}
\hline \hline & Current $I(\mathrm{~A})$ & Field $B(\mathrm{~T} / \mathrm{m})$ & $\begin{array}{c}\text { Detected } \\
\text { energy }(\mathrm{MeV})\end{array}$ \\
\hline Q1 & 81.91 & 14.58 & \\
Q2 & -244.74 to -252.74 & -12.08 to -11.70 & 5 \\
Q3 & 284.75 & 14.19 & \\
\hline \hline
\end{tabular}

consisting of a $90 \mu \mathrm{m}$ aluminum foil and EBT3 film (with a central detecting energy of $5 \mathrm{MeV}$ ). The detectors were placed in a retractable array to enable consecutive measurements. The triplet was originally set to focus $5 \mathrm{MeV}$ protons onto the detector. The current settings are shown in Table I. By adjusting the current of Q2 with a 2 A interval, the beam profile changes can be detected, as shown in Figure 3, where (a) to (e) are the images from the RCF. Further details on this specific setup and accompanying simulation results can be obtained from ref [21].

Assuming the proton beam is transported from initial position $z_{0}$ to a position $z$, its $\sigma$-matrix can be determined from the transport matrix $R(z)$ and the initial matrix $\sigma\left(z_{0}\right)$. The first term in the $\sigma(z)$ matrix, $\sigma_{11}(z)$, is derived from the measured beam profile (the square of the beam spot width). $R(z)$ here is the matrix of the three quadrupole magnets with different magnetic gradients. Thus the emittance can be calculated as:

$$
\varepsilon_{n, \mathrm{rms}}=\frac{\beta \gamma \sigma_{11}(z)_{\mathrm{rms}}}{\beta_{0} R_{11}^{2}-2 \alpha_{0} R_{11} R_{12}+\gamma_{0} R_{12}^{2}} .
$$

There are three unknown parameters: $\alpha_{0}, \beta_{0}$, and $\gamma_{0}$, the Twiss parameters at the source. To calculate the emittance
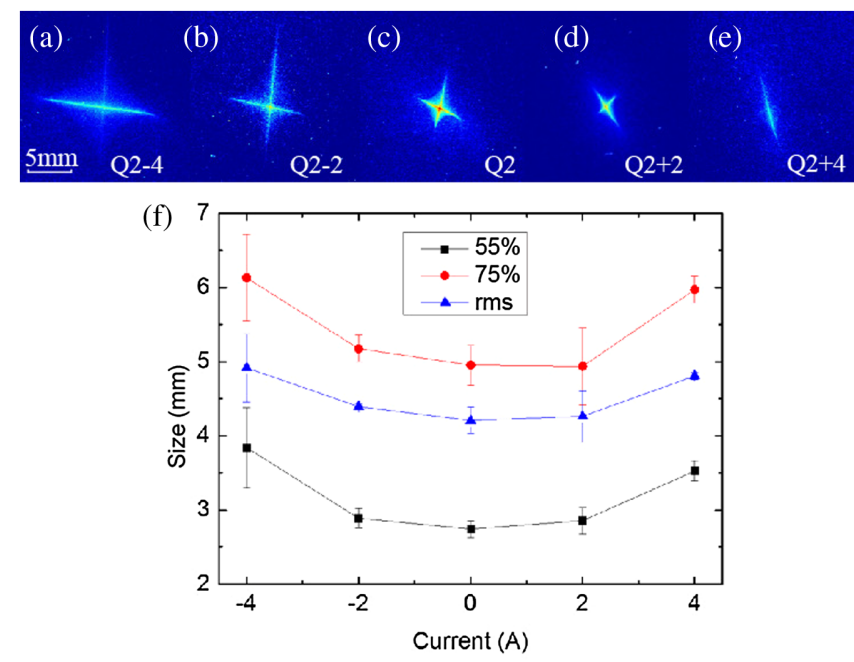

FIG. 3. Proton beam profile as detected by RCF using QST. Plots (a)-(e) show the profile with current shifts of -4 A, -2 A, $0 \mathrm{~A}, 2 \mathrm{~A}$, and $4 \mathrm{~A}$ respectively applied to $\mathrm{Q} 2$, relative to the nominal current of -248.74 A (see Table I). (f) Beam size in the $y$-direction for the same current values. 
therefore requires at least three measurements with different settings. Figure 3(f) shows the variation of the beam size in the $y$-direction corresponding to the different Q2 current settings, where the blue, black, and red curves are the rms width and the widths containing $55 \%$ and $75 \%$ of the protons, respectively. The error bars come from the $13 \%$ fluctuation of the protons per shot [27]. The emittance calculated by Eq. (4) using the least square method is $0.048_{-0.018}^{+0.006} \mathrm{~mm}$ mrad for $5 \mathrm{MeV}$ protons. The error bars are determined by the width containing $55 \%$ and $75 \%$ protons, which have covered the fluctuation of beam size. The value calculated here is at least twice as large as using the pepperpot method. A possible reason for this is the dispersion caused by quadrupole coupled with the large energy spread. There is a $\pm 2 \%$ energy spread for the RCF detector in this experiment, which is ignored in the calculation.

\section{SINGLE-SHOT EMITTANCE MEASUREMENT}

After focusing by the quadrupole triplet, the protons are then dispersed by the dipole magnet according to their energy. The system was set to transport $5 \mathrm{MeV}$ protons. A $100 \mu \mathrm{m}$-thick plastic scintillator (EL-JEN Technology EJ212) coupled with an electron-multiplying charge-coupled device (EMCCD) was placed at the image plane of the dipole. The spatial resolution of the scintillator is $98 \mu \mathrm{m}$, corresponding to an energy resolution of $9.1 \mathrm{keV}$ at $5 \mathrm{MeV}$. Besides the magnetic field, the transport matrix $R(z)$ through the magnet is also energy-dependent. As the quadrupole lens is a point-to-point image system, then if the object changes, the image changes in response. The proton source point is regarded as the beam waist, so $\alpha_{0}$ (which represents the inclination of phase space) is zero. After the dipole magnet, the rms size in the $y$-direction is related to the energy as:

$\sigma_{y, n, \mathrm{rms}}(E)=\sqrt{R_{11}(E)^{2} \sigma_{y 0}^{2}+\left(\frac{\varepsilon_{y, n, \mathrm{rms}}}{\beta \gamma \sigma_{y 0}}\right)^{2} R_{12}(E)^{2}}$,

where $\sigma_{y, n, \mathrm{rms}}(E)$ is the rms beam size in the $y$-direction, $R_{12}$ is designed to be zero for the reference energy, $\left|R_{11}\right|$ acts as the magnification, $\sigma_{y 0}$ is the initial source size, and $\varepsilon_{y, n, \mathrm{rms}}$ is the normalized rms emittance in the $y$-direction. In this experiment, within $\pm 0.2 \mathrm{MeV}$ of the reference energy, the elements of $R$ have a predominantly linear dependence on $E$ with $R_{11}(E)=4.82(E-4.93)-14$ and $R_{12}(E)=162.5(E-4.93)$, in which $E$ is in units of $\mathrm{MeV}$, and $R$ is dimensionless. The offset of the matrix here is smaller than 8 pixels in the EMCCD, which was ignored in this calculation. So the emittance can be calculated by fitting the $\sigma_{y, \text { rms }}(E)$ curve. In this method, energy resolution is included in the transport matrix, so what is measured is the normalized rms emittance in phase space.

One of the single-shot images is shown in Fig. 4(a). The rms beam size in the $y$-direction, $\sigma_{y, n, \text { rms }}(E)$, is shown as the

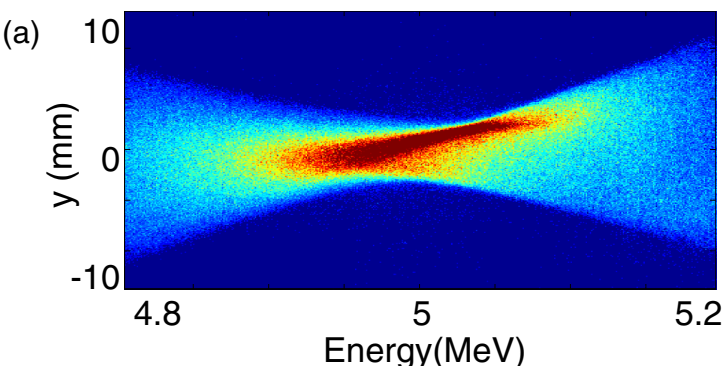

(b)

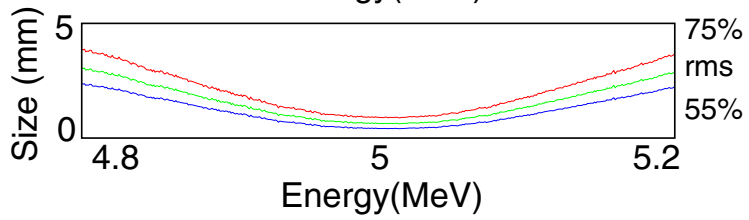

FIG. 4. Single-shot emittance measurements. (a) Example single-shot detected image. (b) Measured beam size variation with energy.

green line of Fig. 4(b). The blue and red lines are determined by the threshold where the profile contains $55 \%$ and $75 \%$ of the protons. The beam profile shows a bow-tie shape due to the energy dispersion [27]. The singleshot emittance measured here is $0.073_{-0.026}^{+0.037} \mathrm{~mm} \mathrm{mrad}$ for $5 \mathrm{MeV}$ protons.

\section{SIMULATION AND DISCUSSION}

Figure 5 summarizes the emittance measurements for the $5 \mathrm{MeV}$ laser-accelerated proton beam, measured at three different positions with the three methods. The solid curves indicate the evolution of emittance as simulated with the program CST [29]. Protons with source size of $50 \mu \mathrm{m}$, energy spread $\pm 2 \%$ (same as the RCF sensitivity) and divergence $\pm 20 \mathrm{mrad}$ were simulated, corresponding to an original emittance $\varepsilon_{n, \text { tr, rms }} \approx \varepsilon_{n, \text { rms }}=0.02 \mathrm{~mm} \mathrm{mrad}$. There is clear growth in $\varepsilon_{n \text {,rms }}$ both inside the triplet and in the drift space. At the scintillator after the dipole magnet,

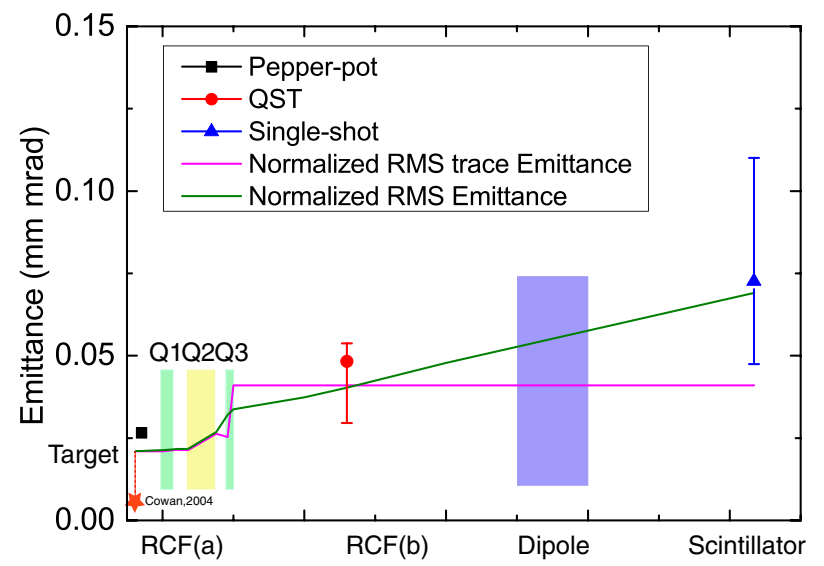

FIG. 5. Emittance values from simulation and experiment. Solid lines are the emittance evolution during transport in simulations. Points with error bars are experimental results. 
$\varepsilon_{n, \text { tr, rms }}$ reaches $0.04 \mathrm{~mm}$ mrad and $\varepsilon_{n, \text { rms }}$ reaches $0.07 \mathrm{~mm}$ $\mathrm{mrad}$, a threefold increase from the original value. The emittance measured by experiments matches the CST simulation well. We show that, for laser accelerated protons, the initial extremely small emittance at the source point (less than $0.004 \mathrm{~mm}$ mrad as measured by Cowan [2]) increases by more than one order of magnitude after a few centimeters of propagation, and remains between $0.04 \mathrm{~mm}$ $\mathrm{mrad}$ to $0.10 \mathrm{mmrad}$ for the subsequent transport. This conclusion is suitable for the measured protons with $\pm 2 \%$ energy spread and $\pm 20 \mathrm{mrad}$ angle to meet with the paraxial condition, which means for the design of a monoenergetic beamline, the laser accelerator can be regarded as the same as the conventional accelerator. However, to fully use the laser accelerated protons with large divergence and broad energy spread, an achromatic beam line with large acceptance angle is recommended. In this condition, the emittance will increased significantly under the double effect of the chromatic aberration and the nonlinear edge field of the magnet components, and must be considered.

\section{CONCLUSIONS}

Emittance is a crucial parameter for describing beam quality, and also determines the beam envelope during transmission. The emittance of laser-driven proton beams have been measured systematically at CLAPA by a variety of different methods. The value of the emittance is measured at the order of $0.1 \mathrm{~mm}$ mrad for $5 \mathrm{MeV}$ protons. Emittance growth caused by the wide energy spread and beam divergence is shown in both simulation and experiment, which cannot be neglected for future achromatic laser proton beamline designs.

\section{ACKNOWLEDGMENTS}

This work was supported by the National Natural Science Foundation of China (Grants No. 11975037, No. 11475010, No. 11921006, No. 11535001), the ITER-CHINA program (Grant No. 2015 GB120001), and the National Grand Instrument Project (No. SQ2019YFF010006).

[1] A. Higginson, R. Gray, M. King, R. Dance, S. Williamson, N. Butler, R. Wilson, R. Capdessus, C. Armstrong, J. Green et al., Near-100 MeV protons via a laser-driven transparency-enhanced hybrid acceleration scheme, Nat. Commun. 9, 724 (2018).

[2] T. E. Cowan, J. Fuchs, H. Ruhl, A. Kemp, P. Audebert, M. Roth, R. Stephens, I. Barton, A. Blazevic, E. Brambrink et al., Ultralow Emittance, Multi-MeV Proton Beams from a Laser Virtual-Cathode Plasma Accelerator, Phys. Rev. Lett. 92, 204801 (2004).

[3] F. Nürnberg, M. Schollmeier, E. Brambrink, A. Blažević, D. Carroll, K. Flippo, D. Gautier, M. Geissel, K. Harres,
B. Hegelich et al., Radiochromic film imaging spectroscopy of laser-accelerated proton beams, Rev. Sci. Instrum. 80, 033301 (2009).

[4] M. Borghesi, A. J. Mackinnon, D. H. Campbell, D. G. Hicks, S. Kar, P. K. Patel, D. Price, L. Romagnani, A. Schiavi, and O. Willi, Multi-MeV Proton Source Investigations in Ultraintense Laser-Foil Interactions, Phys. Rev. Lett. 92, 055003 (2004).

[5] B. Dromey, M. Coughlan, L. Senje, M. Taylor, S. Kuschel, B. Villagomez-Bernabe, R. Stefanuik, G. Nersisyan, L. Stella, and J. Kohanoff, Picosecond metrology of laser-driven proton bursts, Nat. Commun. 7, 10642 (2016).

[6] M. Barberio, M. Scisciò, S. Vallières, F. Cardelli, S. Chen, G. Famulari, T. Gangolf, G. Revet, A. Schiavi, M. Senzacqua et al., Laser-accelerated particle beams for stress testing of materials, Nat. Commun. 9, 372 (2018).

[7] M. Barberio, M. Scisciò, S. Vallières, S. Veltri, A. Morabito, and P. Antici, Laser-Generated Proton Beams for High-Precision Ultra-Fast Crystal Synthesis, Sci. Rep. 7, 12522 (2017).

[8] A. Y. Faenov, T. Pikuz, Y. Fukuda, M. Kando, H. Kotaki, T. Homma, K. Kawase, T. Kameshima, A. Pirozhkov, A. Yogo et al., Submicron ionography of nanostructures using a femtosecond-laser-driven-cluster-based source, Appl. Phys. Lett. 95, 101107 (2009).

[9] S. Kraft, C. Richter, K. Zeil, M. Baumann, E. Beyreuther, S. Bock, M. Bussmann, T. Cowan, Y. Dammene, W. Enghardt et al., Dose-dependent biological damage of tumour cells by laser-accelerated proton beams, New J. Phys. 12, 085003 (2010).

[10] M. Roth, T. E. Cowan, M. H. Key, S. P. Hatchett, C. Brown, W. Fountain, J. Johnson, D. M. Pennington, R. A. Snavely, S. C. Wilks et al., Fast Ignition by Intense Laser-Accelerated Proton Beams, Phys. Rev. Lett. 86, 436 (2001).

[11] A. B. Zylstra, J. A. Frenje, P. E. Grabowski, C. K. Li, G. W. Collins, P. Fitzsimmons, S. Glenzer, F. Graziani, S. B. Hansen, S. X. Hu et al., Measurement of Charged-Particle Stopping in Warm Dense Plasma, Phys. Rev. Lett. 114, 215002 (2015).

[12] P. Patel, A. Mackinnon, M. Key, T. Cowan, M. Foord, M. Allen, D. Price, H. Ruhl, P. Springer, and R. Stephens, Isochoric Heating of Solid-Density Matter with an Ultrafast Proton Beam, Phys. Rev. Lett. 91, 125004 (2003).

[13] J.-G. Zhu, K. Zhu, L. Tao, Y.-X. Geng, C. Lin, W.-J. Ma, H.-Y. Lu, Y.-Y. Zhao, Y.-R. Lu, J.-E. Chen et al., Beam line design of compact laser plasma accelerator, Chin. Phys. Lett. 34, 054101 (2017).

[14] M. Nishiuchi, I. Daito, M. Ikegami, H. Daido, M. Mori, S. Orimo, K. Ogura, A. Sagisaka, A. Yogo, A. Pirozhkov et al., Focusing and spectral enhancement of a repetitionrated, laser-driven, divergent multi-MeV proton beam using permanent quadrupole magnets, Appl. Phys. Lett. 94, 061107 (2009).

[15] F. Schillaci, L. Pommarel, F. Romano, G. Cuttone, M. Costa, D. Giove, M. Maggiore, A. Russo, V. Scuderi, V. Malka et al., Characterization of the ELIMED Permanent Magnets Quadrupole system prototype with laser-driven proton beams, J. Instrum. 11, T07005 (2016). 
[16] M. Schollmeier, S. Becker, M. Geißel, K. A. Flippo, A. Blažević, S. A. Gaillard, D. C. Gautier, F. Grüner, K. Harres, M. Kimmel et al., Controlled Transport and Focusing of Laser-Accelerated Protons with Miniature Magnetic Devices, Phys. Rev. Lett. 101, 055004 (2008).

[17] A. Macchi, M. Borghesi, and M. Passoni, Ion acceleration by superintense laser-plasma interaction, Rev. Mod. Phys. 85, 751 (2013).

[18] M. Roth and M. Schollmeier, Ion acceleration - target normal sheath acceleration, arXiv:1705.10569.

[19] Y.-x. Geng, Q. Liao, Y.-r. Shou, J.-g. Zhu, X.-h. Xu, M.-j. Wu, P.-j. Wang, D.-Y. Li, T. Yang, R.-h. Hu, D.-H. Wang, Y.-Y. Zhao, W. Ma, H.-Y. Lu, C. Lin, and X.-Q. Yan, Generating Proton Beams Exceeding $10 \mathrm{MeV}$ Using High Contrast 60TW Laser, Chin. Phys. Lett. 35, 092901 (2018).

[20] M. Roth, T.E. Cowan, J. Gauthier, J. M. Vehn, M. Allen, P. Audebert, A. Blazevic, E. Brambrink, J. Fuchs, M. Geissel et al., Intense, high-quality ion beams generated by ultra-intense lasers, AIP Conf. Proc. 650, 485 (2002).

[21] M. Wu, J. Zhu, D. Li, T. Yang, Q. Liao, Y. Geng, X. Xu, C. Li, Y. Shou, Y. Zhao, Y. Lu, H. Lu, W. Ma, C. Lin, K. Zhu, and $\mathrm{X}$. Yan, Collection and focusing of laser accelerated proton beam by an electromagnetic quadrupole triplet lens, Nucl. Instrum. Methods Phys. Res., Sect. A 955, 163249 (2020).
[22] M. Zhang, Emittance formula for slits and pepper-pot measurement, Fermi National Accelerator Lab. Technical Report, 1996.

[23] M. G. Minty and F. Zimmermann, Measurement and Control of Charged Particle Beams (Springer Science \& Business Media, New York, 2013).

[24] S. K. Barber, J. van Tilborg, C. B. Schroeder, R. Lehe, H.-E. Tsai, K. K. Swanson, S. Steinke, K. Nakamura, C. G. R. Geddes, C. Benedetti et al., Measured Emittance Dependence on the Injection Method in Laser Plasma Accelerators, Phys. Rev. Lett. 119, 104801 (2017).

[25] K. Floettmann, Some basic features of the beam emittance, Phys. Rev. Accel. Beams 6, 034202 (2003).

[26] M. Migliorati, A. Bacci, C. Benedetti, E. Chiadroni, M. Ferrario, A. Mostacci, L. Palumbo, A. R. Rossi, L. Serafini, and P. Antici, Intrinsic normalized emittance growth in laser-driven electron accelerators, Phys. Rev. Accel. Beams 16, 011302 (2013).

[27] J. Zhu, M. Wu, Q. Liao, Y. Geng, K. Zhu, C. Li, X. Xu, D. Li, Y. Shou, T. Yang et al., Experimental demonstration of a laser proton accelerator with accurate beam control through image-relaying transport, Phys. Rev. Accel. Beams 22, 061302 (2019).

[28] J. F. Ziegler, SRIM-2003, Nucl. Instrum. Methods Phys. Res., Sect. B 219-220, 1027 (2004).

[29] H. Spachmann and U. Becker, Electron gun simulation with CST PARTICLE STUDIO, Nucl. Instrum. Methods Phys. Res., Sect. A 558, 50 (2006). 